\title{
Surgical Options for Localized and Advanced Gastrointestinal Stromal Tumors
}

\author{
TIMOTHY L. FRANKEL, MD, ALFRED E. CHANG, MD, ${ }^{*}$ AND SANDRA L. WONG, MD MS \\ Division of Surgical Oncology, University of Michigan, Ann Arbor, Michigan
}

\begin{abstract}
The development of imitinab has led to a revolution in the management of gastrointestinal stromal tumors (GIST), but surgical resection remains the cornerstone of treatment for patients with localized disease. The principles to surgical treatment of GIST include careful handling of tissues to prevent tumor rupture and resection to negative margins without the need for wide excision. Minimally invasive techniques have proven equally efficacious provided appropriate oncologic resections are performed.
\end{abstract}

J. Surg. Oncol. 2011;104:882-887. (C) 2011 Wiley Periodicals, Inc.

KEY WoRDS: Gastrointestinal stromal tumors; surgery; imitinab; Neoadjuvant; laparoscopy

\section{INTRODUCTION}

Gastrointestinal stromal tumors (GIST) only comprise a small minority of all gastrointestinal malignancies. Though they represent the most common mesenchymal tumor of the GI tract, there are only 5,000-6,000 new cases per year in the United States [1]. Over the past decade, GIST have become the paradigm of targeted molecular therapy. Historically, these rare tumors were variously classified as leiomyosarcoma or leiomyoblastoma, leiomyomas, or GI autonomic nerve tumors. Cytotoxic chemotherapeutics were ineffective in controlling disease and resection was the only effective treatment for GIST. Metastatic and recurrent disease were commonly seen and, in those scenarios, survival was poor. The identification of a mutation in the juxtamembrane domain of the c-kit receptor kinase in up to $90 \%$ of tumors has led to widespread application of imitinab mesylate, a small molecule capable of occupying the ATP binding site of tyrosine kinase thereby inhibiting phosphorylation of downstream signaling motifs.

While improved diagnostics and the development of effective small molecule inhibitors, which inhibit the KIT proto-oncogene mutations have led to revolution in the diagnosis and management of GIST, resection remains the cornerstone of treatment for patients with localized disease. Imitinab is the first line therapy for metastatic and recurrent GIST and can often be used in conjunction with surgical resection to produce long-term survival. A multidisciplinary approach to the management of GIST combines radiologic, endoscopic, pathologic evaluations, and treatment with resection and targeted therapy with tyrosine kinase inhibitors (TKI) [2].

\section{SURGICAL TREATMENT Of PRIMARY GIST}

\section{Diagnostic Considerations}

Patients with GIST can present with a variety of symptoms dictated primarily by their size and location at diagnosis. Small lesions are often found incidentally during endoscopy or laparotomy for other indications. GIST generally produce symptoms from mass effect though may be the source of significant GI bleeding. Larger tumors tend to be more symptomatic though small tumors are also associated with nausea, pain, early satiety, and bleeding (Figure 1). GIST may ulcerate in to the GI lumen, resulting in subclinical bleeding with microcytic anemia as a presenting symptom or in some cases, causing significant hematochezia. If tumors are particularly large, abdominal distention or a palpable mass may be noted. GIST occurring in or near narrow regions of the GI tract, such as the gastroesophageal junction or the pylorus may present with obstruction [3].

Initial workup should include a thorough physical exam and detailed history followed by cross sectional imaging using computed tomography to both assess the extent of the primary tumor and evaluate potential sites of metastatic disease, most commonly the liver, omentum, or peritoneum. The origin of the tumor is typically within the muscularis propria, so focal mural thickening can be seen at the site of origin. Lymphatic metastases are rare and unlike other sarcomas, spread to lung is uncommon. Features on magnetic resonance imaging can be helpful for diagnosis. GIST typically enhance with gadolinium contrast administration and are noted to have low signal intensity on T1-weighted images and high signal intensity on T2-weighted images. ${ }^{18}$ Flourodeoxyglucose positron emission tomography (FDG-PET) can be used to evaluate GIST since the tumors tend to be FDG avid. While PET can detect and characterize suspicious distant lesions, its more common use is in evaluating response to TKI therapy. PET before or after resection is not routinely used. Endoscopic ultrasound can be helpful in better localizing tumors and establishing a diagnosis, often by ultrasonographic confirmation of intramural tumor origin.

Because tumors are fragile and often hypervascular, biopsy is not routinely recommended for diagnosis due to risk of capsular perforation, intraperitoneal seeding of tumor cells, and bleeding. While endoscopically- or radiologically-guided biopsies can be performed accurately, the submucosal location of many tumors often preclude accurate sampling. Often, GIST have necrotic centers, limiting the diagnostic utility of fine needle aspirates or even core needle biopsies [4]. However, biopsies may be helpful in select cases such as when there is diagnostic uncertainty such as cases in which lymphoma or other processes are a diagnostic consideration, or when location prompts suspicion for other sarcoma subtypes (Figure 2) and situations where tumors are marginally resectable or primarily unresectable [5].

*Correspondence to: Alfred E. Chang, MD, Division of Surgical Oncology, University of Michigan, 3303 Cancer Center, 1500 E. Medical Center Drive, Ann Arbor, MI 48109. Fax: 7346479647.

E-mail: aechang@umich.edu

Received 3 January 2011; Accepted 25 January 2011

DOI $10.1002 /$ jso. 21892

Published online 4 March 2011 in Wiley Online Library (wileyonlinelibrary.com). 


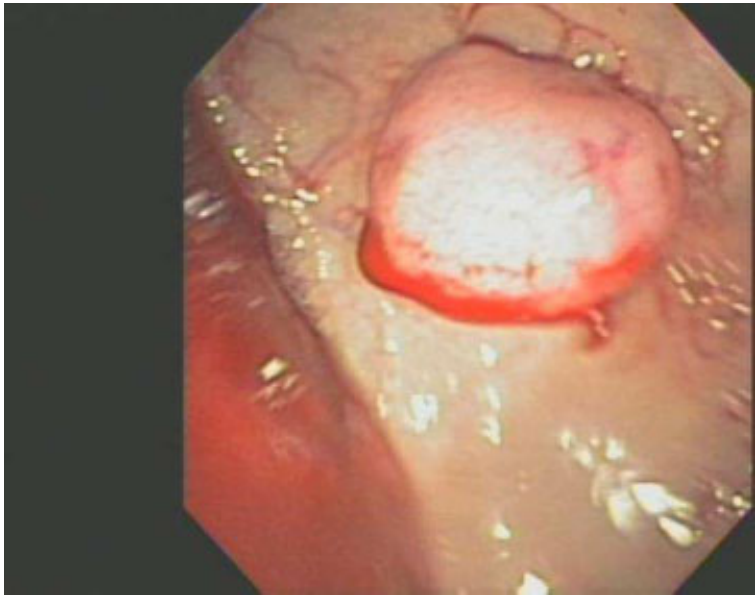

Fig. 1. This is a case of a 64-year old man who presented with massive hematemesis, tachycardia, hypotension, and severe anemia. $\mathrm{He}$ required massive transfusion and was found to have a submucosal mass with spurting bleeding in the gastric fundus. He underwent emergent resection of the mass with wedge gastrectomy for a histologically benign spindle cell gastrointestinal stromal tumor measuring $3.8 \mathrm{~cm}$ in size. [Color figure can be viewed in the online issue, available at wileyonlinelibrary.com.]

\section{Principles of Surgical Treatment}

Once a diagnosis of GIST is confirmed and metastatic disease excluded, an operative strategy is formulated based on the size and location of the tumor [6]. Controversy exists regarding treatment of small lesions $(<2 \mathrm{~cm})$ incidentally identified during endoscopy, cross sectional imaging, or surgical exploration for other indications. Most would agree that a small tumor found at the time of laparotomy or laparoscopy should be removed if in a location that would not cause

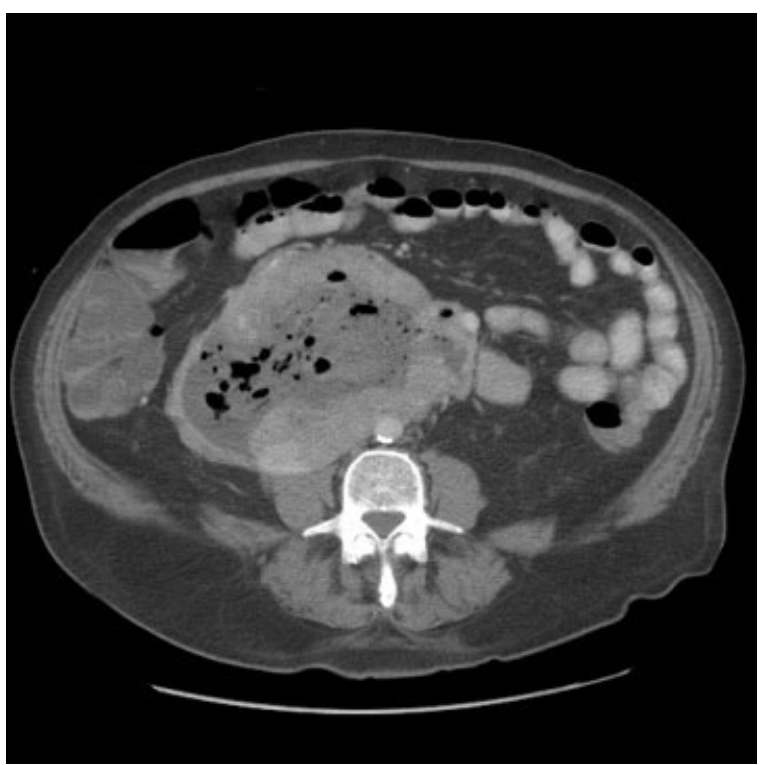

Fig. 2. Cross-sectional imaging demonstrates an $18 \times 11 \mathrm{~cm}$ mass nearly encasing the IVC and aorta, with central low attenuation features and gas. Differential diagnosis included leiomyosarcoma of the IVC though biopsy confirmed a diagnosis of gastrointestinal stromal tumor. undo morbidity. When GISTs are identified during routine endoscopy, decision-making may be more difficult due to the lack of data regarding growth rate and metastatic potential of small tumors. Endoscopic features are unable to predict tumor behavior. In a recent series of 23 patients found to have small GIST on routine endoscopy, only three had increase in size of their lesion on follow-up exam [7]. These patients underwent subsequent surgical resection without evidence of metastatic spread. Although sufficient data on management of small incidentally discovered GIST are lacking, a reasonable strategy can include surveillance in 6-12 months. For lesions $>2 \mathrm{~cm}$, surgical resection is the principle treatment and provides the only means for cure.

At time of operation, care should be taken to thoroughly evaluate the abdomen for metastatic disease paying particular attention to the liver and peritoneum, the most common sites of disease spread. Peritoneal disease is often missed on preoperative imaging and suspicious lesions should be resected if possible or biopsied to stage the patient if too extensive for complete resection. Intraoperatively, care should be taken when handling the lesion to ensure rupture does not occur and the tumor pseudocapsule stays intact. This prevents excessive bleeding and peritoneal dissemination of tumor cells. Unlike adenocarcinomas which tend to infiltrate surround tissue, GISTs have an extraluminal and exophytic growth pattern often with a narrow base of attachment. Because of this, surgical resection to microscopically clear margins is often easily achieved with minimal removal of uninvolved tissue. As with other sarcomas, GISTs tend not to invade surrounding structures, but rather displace them. Some tumors often lift away from surrounding structures without the need for extended resection. This often means favoring a segmental as opposed to an anatomic resection.

Other tumors may become densely adherent to neighboring organs such as the liver or spleen. In these instances, en bloc resection is preferred to prevent capsular disruption. In some cases, there is both intra- and extra-luminal extension of tumor, requiring more a more extensive resection for clearance of disease. Formal lymphadenectomy is not required. If enlarged nodes are found at the time of resection, these should be resected with the primary lesion.

\section{Esophagus}

GISTs of the esophagus are quite rare comprising $<5 \%$ of the overall incidence [8]. Because of this, little data exists regarding the appropriate management of these lesions. Often confused for the more common leiomyomas, esophageal GISTs present with dysphagia or bleeding. Indistinguishable on endoscopy and swallow study, these lesions require biopsy and proper immunohistochemical staining prior to surgical intervention. Open or thoracoscopic enucleation remains the treatment of choice for bengin leimyomas, but this is not sufficient for the treatment of GIST. Enucleation often leaves behind a tumor laden pseudocapsule contributing to local recurrence. Strategies for resection vary with location and more importantly size of the tumor. Small lesions $(<2 \mathrm{~cm})$ can occasionally be treated with a transmural wide excision and repair, assuming an R0 margin is possible [9]. In a series of four patients, Blum et al. [10] report local resection of esophageal GIST with primary closure of the defect. At a median follow-up of 3 years, two patients had recurrent disease, one distant and one at the resection site. The latter was treated with an esophagectomy and remained disease free at follow-up. Care must be taken not to narrow the esophageal lumen which can result in a poor long-term outcome. For larger lesions, especially those located near the GE junction, a formal esophagectomy with either an intrathoracic or cervical esophago-gastrostomy is needed [11]. In patients with large tumors, neoadjuvant imitinab mesylate should be considered to decrease tumor size and improve the probability of a satisfactory resection. 


\section{Stomach}

The stomach is the most common site for GISTs with gastric tumors comprising $70 \%$ of the overall incidence. Patients often present with GI bleeding, but can also present with gastric obstruction when tumors become large or occur near the gastro-esophageal junction or pylorus. They are best worked up with upper endoscopy with endoscopic ultrasound to confirm intramural origin. Biopsy is not required to make the diagnosis of GIST. Small tumors are occasionally found during endoscopy for other indications such as GERD or ulcer disease. Gastric tumors tend to be more indolent than those in the intestine or esophagus with smaller tumors rarely leading to metastatic disease or death [12]. When matched by size and mitotic rate, non-gastric location is an independent poor prognostic factor [13]. In a series of 1765 patients, the overall tumor specific mortality was $17 \%$ and only $2 \%$ for patients with tumors $<10 \mathrm{~cm}$.

Resection strategy for gastric GIST depends primarily on the location of the lesion within the stomach. As with all GISTs, the operative goal is segmental resection with clear margins while limiting potential tumor rupture by violating the tumor pseudocapsule. Lesions located on the greater curve can most often be managed with a wedge or sleeve-type resection. After inspection for metastatic disease, the short gastric vessels are divided separating the stomach from the spleen. An area proximal and distal to the tumor is then identified and resection performed using a stapler. Care must be taken not to significantly narrow the stomach leading to a functional obstruction. This may be more common with large lesions and those located at the incisura angularis along the lesser curve though wedge resections can still be performed depending on anatomic contraints. Antral lesions pose a similar problem as wedge resection can result in gastric outflow obstruction. Unless the tumor is small, most would advocate a distal gastrectomy with gastrojejunal or gastroduodenal reconstruction.

Proximal lesions on the lesser curve or close proximity to the GE junction may also be difficult to treat, especially if there is a fairly broad base of origin. Small lesions may be excised with closure of the resultant defect ensuring the gastric inflow is not compromised [14]. Even tumors near the GE junction can be handled with local resections (Figure 3). For larger tumors, subtotal gastrectomy may be required to remove the tumor. Despite some technical challenges, an $\mathrm{R} 0$ resection is possible in a majority of cases $[15,16]$.

Unlike adenocarcinoma, omentectomy or extended lymphadenectomy is not required unless obviously involved with disease. Occasionally, large tumors can become adherent to adjacent structures, requiring en bloc omentectomy, splenectomy, or distal pancreatectomy to ensure capsule integrity. In a single institution review of 37 patients undergoing resection of large $(>10 \mathrm{~cm})$ GISTs, $51 \%$ of patients required removal of adjacent organs [17]. These patients had a longer operative time and tended to have a poorer overall prognosis.

\section{Duodenum}

Although the small intestine represents the second most common site for GIST, duodenal origin remains rare at less $<5 \%$ the overall incidence [18]. While a majority of patients present with bleeding, large tumors can lead to GI or pancreatico/biliary obstruction. The best modality for diagnosis is endoscopy, but this can prove difficult for obstructing lesions, or those occurring in the distal duodenum (D4). Similar to gastric GIST, the resection strategy depends on size and location of the primary tumor as well as distance from the ampulla. Small tumors in D1 or D2 that do not directly involve the ampulla can be treated with wide excision and primary closure with satisfactory results $[18,19]$. Lesions in D3 or D4 are best treated with segmental resection with restoration of GI continuity via a duodeno-

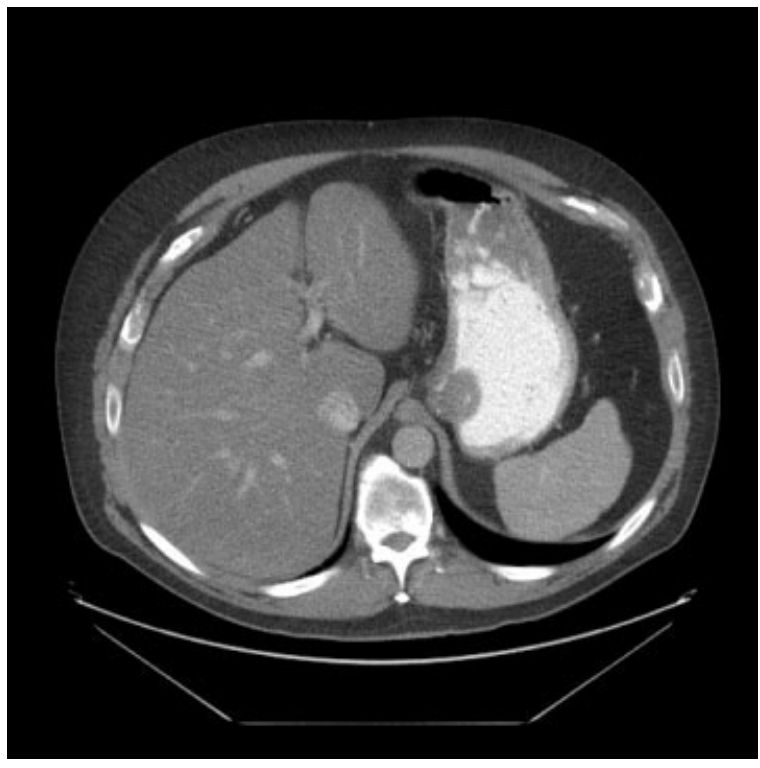

Fig. 3. Cross-sectional imaging with oral contrast demonstrates a $3.9 \times 3.3 \mathrm{~cm}$ well-demarcated mass at the gastroesophageal junction/proximal cardia with protrusion into the gastric lumen. Wedge gastrectomy with modified Nissen fundoplication was used to accomplish complete resection of this GIST.

jejunostomy. For large duodenal tumors or those close to or involving the ampulla, a pancreaticoduodenectomy is often needed for complete resection. In a large series of duodenal GISTs reported by Mitettinen et al. [18], 156 tumors were treated with enucleation $(n=15)$, wide excision $(n=21)$, segmental resection $(n=48)$, and pancreaticoduodenectomy $(\mathrm{n}=21)$. Unfortunately, data was not presented on the adequacy of resection or recurrence rate by type of resection. Yang et al. [20] reported a series of 21 patients undergoing local resection $(n=12)$ or pancreaticoduodencetomy $(n=9)$. All patients were resected to negative microscopic margins and only one experienced recurrent disease. Although limited data exists, there have been reports of neoadjuvant treatment with imatinib mesylate leading to reduction in tumor size allowing for a more limited duodenal resection [14].

\section{Jejunum and Ileum}

The small intestine is the second most common location for GISTs, comprising $20 \%$ of the overall incidence [21]. As with duodenal GISTs, GI bleeding is the most common presenting symptom followed by obstruction [22]. Diagnosis can be difficult, but visualization of the lesion is often possible via push endoscopy or video capsule endoscopy [23]. The former is the preferred technique as biopsy is not possible when the capsule is used and entrapment of the device has been reported [24]. In the largest published series of 906 small intestinal GISTs collected at the Armed Forces Institute of Pathology, there was a higher proportion of lesions found in the jejunum than the ileum [22]. A majority of patients in that series (64\%) presented with tumors over $5 \mathrm{~cm}$ with $28 \%$ measuring $>10 \mathrm{~cm}$. The same surgical principles of negative margins and prevention of tumor rupture apply to intestinal GISTs. This is best accomplished by segmental small bowel resection with primary anastomosis. Proximal jejunal lesions near the root of the mesentery occasionally require segmental resection with a duodenal-jejunal anastomosis [14]. Multiple studies have concluded that small intestinal GISTs are more 
malignant than those found in the stomach. They tend to present with a larger size [12] and more mitotic bodies [22]. Irrespective of these two important factors, small intestinal location is associated with a poorer prognosis [25]. In a large study from Memorial Sloan Kettering Cancer Center of 127 patients with GISTs, intestinal location was an independent predictor of recurrence (HR 3.3, when gastric location was used as a reference) [26].

\section{Colon and Rectum}

Due to its rare occurrence ( $<5 \%$ of overall incidence), little data exists regarding the presentation and management of GISTs occurring in the colon and rectum. The most common presenting symptom is bleeding, with a near equal number of patients presenting with abdominal pain [27]. Obstruction occurs less often than in other GI locations due to the relatively large luminal diameter. Because a large number of patients present with pain as their only symptom, diagnosis is often made using cross sectional imaging. If confirmation of location or a tissue biopsy is needed, colonoscopy should be performed. A study of 17 patients in Taiwan reported a higher incidence of distal lesions with 58\% arising from the rectum and only $18 \%$ in the ascending colon [27]. Colonic GISTs can typically be treated with segmental resection and primary anastomosis. Unlike colonic adenocarcinoma, formal lymphadenectomy is not needed, unless obvious nodal involvement is identified.

Resection of rectal GISTs is more difficult and is often associated with greater morbidity. They often present with a bleeding and perineal pain and are often large in size. Because of the confined space of the pelvis, tumors are often densely adherent to the pelvic floor musculature making $\mathrm{R} 0$ resection challenging. If tumors can be freed from surrounding tissues and sufficient distance from the anal sphincter exists, a low resection if end to end anastomosis is possible. A formal mesorectal excision is unnecessary and often leads to increased morbidity by damaging the autonomic nerves. For lesions in the lower rectum, and abdominoperineal resection is often needed. If the tumor is unable to be separated from pelvic structures, a pelvic exenteration is undertaken. Despite aggressive surgical approaches, the rate of positive margins in rectal GISTs approaches $40 \%$.

\section{LAPAROSCOPY}

In the past 10 years, laparoscopic approaches to GIST have gained popularity due the technical ability to perform complete resections in a minimally invasive fashion. With appropriate handling of the tumor, laparoscopic approaches are safe and effective in selected cases. Although no randomized trials comparing open versus laparoscopically resected tumors exist, reports from multiple small series highlight the safety and feasibility of this approach [2]. Novitsky et al. [28] reported on 50 consecutive patients undergoing laparoscopic resection of GISTs. There were no instances of conversion to open procedure and $96 \%$ of patients remained tumor free at 36 months. In a similar study, Lai et al. [29] reported on 28 patients with gastric GISTs undergoing minimally invasive resection. At a mean follow-up of 43 months, there were no documented recurrences. It is important to follow the same surgical principles of laparotomy when performing laparoscopic GIST resections.

Tumor manipulation should be minimized to reduce the risk of rupture and tumor spread. All specimens should be removed in protective bags or pouches to prevent unintended rupture and port site seeding. There should be little hesitation to convert to an open technique if failure to do so will result in unsatisfactory oncologic outcome or untoward outcome for the patient. Some have advocated a hand assisted technique when dealing with larger tumors $(>5 \mathrm{~cm})$ or when adherence to surrounding structures is encountered [30].

\section{Neoadjuvant Therapy}

The role of preoperative imatinib in the treatment of GIST remains unknown. In many patients with large tumors located in difficult to resect areas, neoadjuvant imatinib can lead to reduction in tumor size making surgical resection both feasible and safe (Figure 4). Furthermore, the risk of tumor rupture during surgical manipulation is reduced after a significant tumor response. In a study of 36 patients with difficult to resect tumors, preoperative administration of imatinib resulted improved ability to completely excise tumors with substantially decreased need for removal of surrounding organs [31]. In a second smaller study by Fiore et al.[32], 15 patients with extensive tumor burden or high perioperative risk underwent
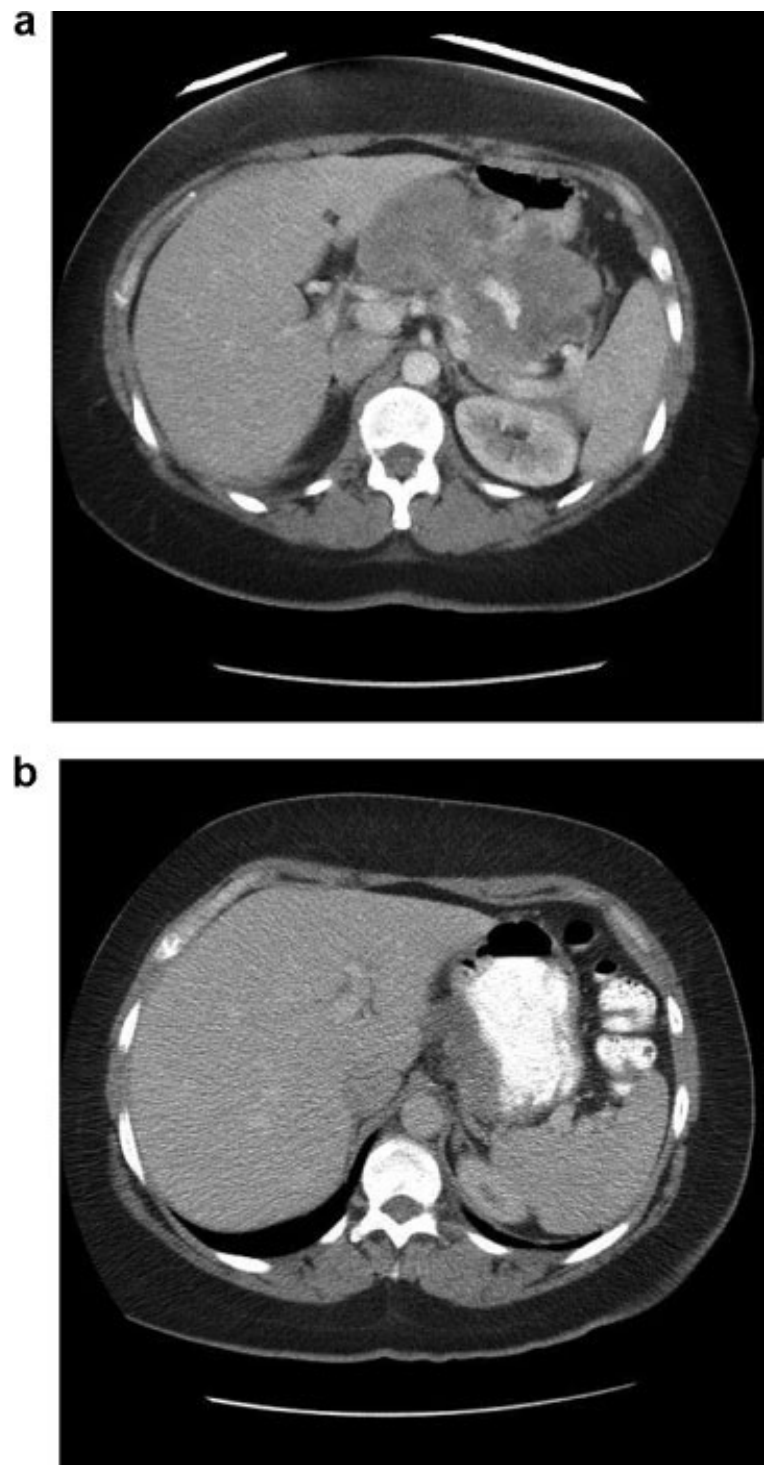

Fig. 4. CT imaging (4a) shows a large gastrointestinal stromal tumor measuring $11.5 \times 7.4 \times 10$ centimeters along the lesser curvature of her stomach. This extended to the left lobe of the liver and the body and tail of the pancreas. The patient was initiated on neoadjuvant imatinib and dramatic radiologic improvement in GIST tumor size was seen. After stability in interval CT scan (4b), the patient underwent exploration and wedge resection of the lesser curvature of the stomach. The tumor was easily separated from surrounding organs. 
neoadjuvant therapy with imatinib prior to surgical resection. At a mean time of 9 months, all patients demonstrated reduction in tumor size and were able to undergo a more limited resection. Although the sample sizes in these reports are small and prone to recall biases in terms of type of operation performed, they do demonstrate the potential utility of this approach in high-risk patients and tumors.

The only multicenter trial investigating the use of neoadjuvant imatinib is the Radiation Therapy Oncology Group (RTOG) 0312. In this trial, patients with primary resectable or recurrent tumors were given $600 \mathrm{mg}$ of imatinib for 8-12 weeks prior to surgery. Patients who experienced objective response or disease stability were eligible for surgical resection followed by 2 years of adjuvant therapy. When compared with historical controls, 2-year disease-free survival was superior in the patients treated with neoadjuvant therapy. Controversy remains regarding the optimal duration of neoadjuvant therapy. Most agree that surgical resection should occur when TKI induced regression plateaus, which typically occurs at 6-9 months after initiation. Patients should be followed with serial CT scans every 3 months to assess continued tumor size reduction. Surgical intervention should occur after stable disease is noted on two consecutive scans. If it remains unclear whether imitinab is having a biologic effect, PET scans can be helpful by documenting decrease or cessation of tumor activity [33]. If tumors display no size reduction, but become PET negative, most would consider surgical intervention. This highlights a unique difference between GIST and other solid organ malignancies.

While response to chemotherapy or radiation is typically measured by reduction is tumor size such as the Response Evaluation Criteria In Solid Tumors or RECIST, this can be misleading in evaluating response to imatinib. The best method for documenting the efficacy of TKIs is in cessation of biologic activity, not reduction in size. Because of this, Choi et al. [34] suggested a new method for documenting response to therapy using not only reduction in tumor size, but changes in tumor density as measured by CT scan. They found that when tumors demonstrated at least a $10 \%$ reduction in size or a $15 \%$ decrease in density, there was near $100 \%$ correlation with decrease in activity determined by PET scan. Many have adopted these new criteria for evaluating response to therapy. If tumors fail to show response to up front imatinib, second and third line TKIs may be used prior to intervention.

Current NCCN guidelines recommend neoadjuvant therapy for patients with large tumors where resection would cause undo morbidity or functional deficit, and small tumors in difficult to treat areas such as the GE junction or low rectum. The use of neoadjuvant imatinib should be decided on a case by case basis at centers with experience in the treatment of GIST [35].

\section{SURGICAL TREATMENT OF METASTATIC DISEASE}

The principal therapy for patients with metastatic GIST is imatinib. It can lead to tumor regression or stability and has dramatically lengthened survival with this disease. Unfortunately, the development of resistance to this therapy is common with $50 \%$ of patients experiencing tumor growth at 2 years. Because of this, if the opportunity exists to resect all visible disease, it should be taken. This usually involves anatomic or non-anatomic hepatic resections as well as removal of peritoneal deposits. In three recent series reporting results of cytoreductive surgery, gross tumor removal was achieved in $80 \%$ of patients with $50 \%$ of patients deemed microscopically disease free [14]. The timing of surgery remains controversial, but most agree that is should occur at the time of maximal tumor response, but before resistance can develop. A widely accepted strategy is to document cessation of tumor regression on consecutive CAT scans prior to operative intervention. This typically occurs $6-9$ months into therapy.
A second indication for resection of metastatic GIST is in the setting of resistance formation. Because survival in patients depends on imitinab mediated disease stability, resection of tumor deposits that develop resistance to chemotherapy and begin to grow makes oncologic sense. There are two types of disease progression of tyrosine kinase blocking therapy. The first is localized progression where isolated growth of limited numbers of metastatic deposits is seen (Figure 5). The second is diffuse progression where multiple or all lesions show growth. DeMatteo et al. [36], reported that while patients with stable disease of localized progression benefit from operative debulking, there is little improvement in overall survival for patients with diffuse progression.

Timing and treatment of patients with metastatic GIST requires experience and expertise necessitating referral of these patients to

a

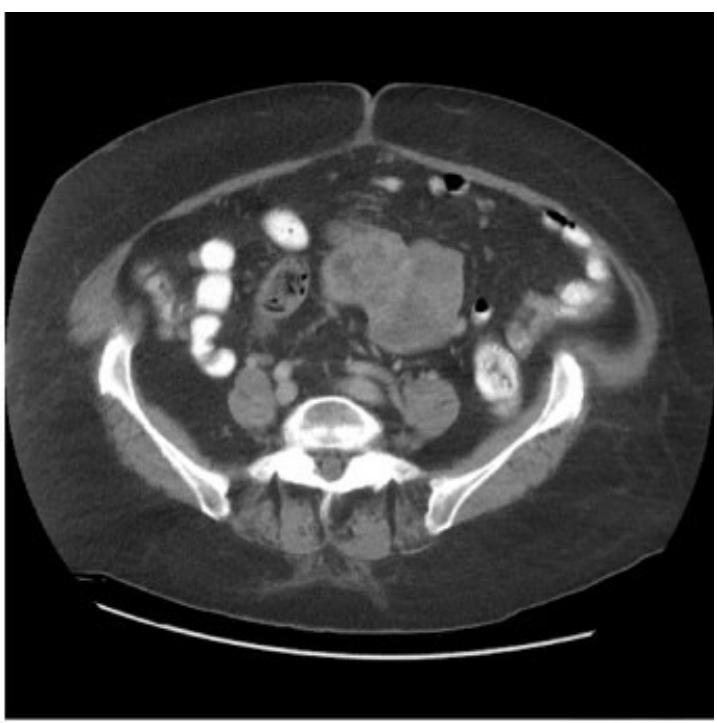

b

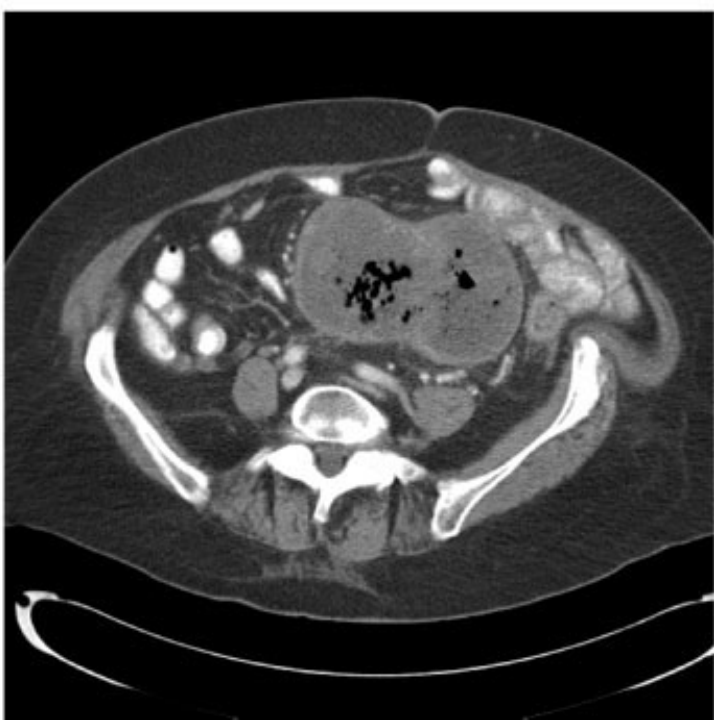

Fig. 5. This is a case of a 71-year old woman diagnosed with metastatic GIST (small bowel primary and innumerable synchronous liver metastases). Disease was stable on imatinib for 3 years (5a), but she was then noted to have lower GI bleeding and progression of disease at the primary site though all the liver lesions were stable (5b). She did not tolerate increased imatinib dosing and second-line sunitinib. With an ongoing transfusion requirement, she underwent resection of a single progressive site of disease with small bowel resection. 
appropriate tertiary care centers. Specific considerations for surgical treatment of recurrent disease are covered extensively elsewhere and are outside the scope of this review.

\section{SUMMARY}

Even with the availability of an effective molecularly targeted agent, the primary treatment modality for localized GIST is surgical resection. Tenets of surgical resection include complete microscopic resection with organ-preserving approaches when possible.

\section{REFERENCES}

1. Fletcher CD, Berman JJ, Corless C, et al.: Diagnosis of gastrointestinal stromal tumors: A consensus approach. Hum Pathol 2002:33:459-465.

2. Kingham TP, DeMatteo RP: Multidisciplinary treatment of gastrointestinal stromal tumors. Surg Clin North Am 2009;89: 217-233.

3. Nilsson B, Bumming P, Meis-Kindblom JM, et al.: Gastrointestinal stromal tumors: The incidence, prevalence, clinical course, and prognostication in the preimatinib mesylate era - A population-based study in western Sweden. Cancer 2005;103:821829.

4. Garcia dePolavieja, Carrasco M, de Juan Ferre A, et al.: Gastrointestinal stromal tumours at present: An approach to burning questions. Clin Transl Oncol 2010;12:100-112.

5. Demetri GD, von Mehren M, Antonescu CR, et al.: NCCN Task Force report: Update on the management of patients with gastrointestinal stromal tumors. J Natl Compr Canc Netw 2010; $8: S 1-S 41$ (quiz S42-S44).

6. Casali PG, Jost L, Reichardt P, et al.: Gastrointestinal stromal tumors: ESMO clinical recommendations for diagnosis, treatment and follow-up. Ann Oncol 2008;19:ii35-38.

7. Lok KH, Lai L, Yiu HL, et al.: Endosonographic surveillance of small gastrointestinal tumors originating from muscularis propria. J Gastrointestin Liver Dis 2009;18:177-180.

8. Miettinen M, Sarlomo-Rikala M, Sobin LH, et al.: Esophageal stromal tumors: A clinicopathologic, immunohistochemical, and molecular genetic study of 17 cases and comparison with esophageal leiomyomas and leiomyosarcomas. Am J Surg Pathol 2000;24:211-222.

9. Hueman MT, Schulick RD: Management of gastrointestinal stromal tumors. Surg Clin North Am 2008;88:599-614 vii.

10. Blum MG, Bilimoria KY, Wayne JD, et al.: Surgical considerations for the management and resection of esophageal gastrointestinal stromal tumors. Ann Thorac Surg 2007;84: $1717-1723$

11. Gouveia AM, Pimenta AP, Lopes JM, et al.: Esophageal GIST: Therapeutic implications of an uncommon presentation of a rare tumor. Dis Esophagus 2005;18:70-73.

12. Miettinen M, Sobin LH, Lasota J: Gastrointestinal stromal tumors of the stomach: A clinicopathologic, immunohistochemical, and molecular genetic study of 1765 cases with long-term follow-up. Am J Surg Pathol 2005;29:52-68.

13. Miettinen M, Lasota J: Gastrointestinal stromal tumors: Pathology and prognosis at different sites. Semin Diagn Pathol 2006;23:70-83.

14. Gervaz P, Huber O, Morel P: Surgical management of gastrointestinal stromal tumours. Br J Surg 2009;96:567-578.

15. Privette A, McCahill L, Borrazzo E, et al.: Laparoscopic approaches to resection of suspected gastric gastrointestinal stromal tumors based on tumor location. Surg Endosc 2008;22: 487-494.

16. Silberhumer GR, Hufschmid M, Wrba F, et al.: Surgery for gastrointestinal stromal tumors of the stomach. J Gastrointest Surg 2009;13:1213-1219.

17. Goh BK, Goh BK, Kesavan SM, et al.: Outcome after curative resection of large $(>0 \mathrm{r}=10 \mathrm{~cm})$ gastric gastrointestinal stromal tumors: How frequent is adjacent organ involvement and is concomitant distal pancreatectomy necessary? J Gastrointest Surg; 14:607-613.

18. Miettinen M, Kopczynski J, Makhlouf HR, et al.: Gastrointestinal stromal tumors, intramural leiomyomas, and leiomyosarcomas in the duodenum: A clinicopathologic, immunohistochemical, and molecular genetic study of 167 cases. Am J Surg Pathol 2003;27:625-641.

19. Buchs NC, Bucher P, Gervaz P, et al.: Segmental duodenectomy for gastrointestinal stromal tumor of the duodenum. World $\mathrm{J}$ Gastroenterol 2010;16:2788-2792.

20. Yang WL, Yu JR, Wu YJ, et al.: Duodenal gastrointestinal stromal tumor: Clinical, pathologic, immunohistochemical characteristics, and surgical prognosis. J Surg Oncol 2009;100: 606-610.

21. Strickland L, Letson GD, Muro-Cacho CA: Gastrointestinal stromal tumors. Cancer Control 2001;8:252-261.

22. Miettinen M, Makhlouf H, Sobin LH, et al.: Gastrointestinal stromal tumors of the jejunum and ileum: A clinicopathologic, immunohistochemical, and molecular genetic study of 906 cases before imatinib with long-term follow-up. Am J Surg Pathol 2006;30:477-489.

23. Lin MB, Yin L, Li JW, et al.: Double-balloon enteroscopy reliably directs surgical intervention for patients with small intestinal bleeding. World J Gastroenterol 2008;14:19361940 .

24. Li F, Gurudu SR, De Petris G, et al.: Retention of the capsule endoscope: A single-center experience of 1000 capsule endoscopy procedures. Gastrointest Endosc 2008;68:174-180.

25. Martin J, Poveda A, Llombart-Bosch A, et al.: Deletions affecting codons 557-558 of the c-KIT gene indicate a poor prognosis in patients with completely resected gastrointestinal stromal tumors: A study by the Spanish Group for Sarcoma Research (GEIS). J Clin Oncol 2005;23:6190-6198.

26. Dematteo RP, Gold JS, Saran L, et al.: Tumor mitotic rate, size, and location independently predict recurrence after resection of primary gastrointestinal stromal tumor (GIST). Cancer 2008; 112:608-615.

27. Chen CW, Wu CC, Hsiao CW, et al.: Surgical management and clinical outcome of gastrointestinal stromal tumor of the colon and rectum. Z Gastroenterol 2008;46:760-765.

28. Novitsky YW, Kercher KW, Sing RF, et al.: Long-term outcomes of laparoscopic resection of gastric gastrointestinal stromal tumors. Ann Surg 2006;243:738-745 (discussion 745-747).

29. Lai IR, Lee WJ, Yu SC: Minimally invasive surgery for gastric stromal cell tumors: Intermediate follow-up results. J Gastrointest Surg 2006;10:563-566

30. Raut CP, Ashley SW: How I do it: Surgical management of gastrointestinal stromal tumors. J Gastrointest Surg 2008;12: 1592-1599.

31. P. Hohenberger OO, Licht T, Dimitrakopoulou-Strauss A, et al.: Neoadjuvant imatinib and organ preservation in locally advanced gastrointestinal stromal tumors (GIST). J Clin Oncol 2009;27:15s

32. Fiore M, Palassini E, Fumagalli E, et al.: Preoperative imatinib mesylate for unresectable or locally advanced primary gastrointestinal stromal tumors (GIST). Eur J Surg Oncol 2009;35:739745.

33. Abhyankar SA, Nair N: Highlighting the role of FDG PET scan in early response assessment of gastrointestinal stromal tumor treated with imatinib mesylate. Clin Nucl Med 2008;33:213-214.

34. Choi H, Charnsangavej C, Faria SC, et al.: Correlation of computed tomography and positron emission tomography in patients with metastatic gastrointestinal stromal tumor treated at a single institution with imatinib mesylate: Proposal of new computed tomography response criteria. J Clin Oncol 2007;25: $1753-1759$.

35. Raut CP, DeMatteo RP: Prognostic factors for primary GIST: Prime time for personalized therapy? Ann Surg Oncol 2008;15:4-6.

36. DeMatteo RP, Maki RG, Singer S, et al.: Results of tyrosine kinase inhibitor therapy followed by surgical resection for metastatic gastrointestinal stromal tumor. Ann Surg 2007;245: $347-352$. 